\title{
A Novel Technique for the Measurement of CBF and CBV with Robot-Arm-Mounted Flat Panel CT in a Large-Animal Model
}

\author{
O. Beuing, A. Boese, Y. Kyriakou, Y. Deuerling-Zengh, B. Jöllenbeck, C. Scherlach, A. Lenz, S. Serowy, S. Gugel, G. Rose, and M. Skalej
}

\begin{abstract}
BACKGROUND AND PURPOSE: Endovascular therapy is an emerging treatment option in patients with acute ischemic stroke and especially in cases presenting late after symptom onset. Information about remaining viable tissue as measured with perfusion imaging is crucial for proper patient selection. The aim of this study was to investigate whether perfusion imaging with C-arm CT in the angiography suite is feasible and provides measurements comparable with ones made by CTP.
\end{abstract}

MATERIALS AND METHODS: The MCA was occluded surgically in 6 sheep. Perfusion studies were performed before surgery, immediately after, and at 3 hours after MCA occlusion by using a robotic flat panel detector $\mathrm{C}$-arm angiographic system. For comparison, conventional CTP was performed at the same time points. Two different protocols with the $\mathrm{C}$-arm CT were tested. Images were analyzed by 2 readers with regard to the presence and size of perfusion abnormalities.

RESULTS: With C-arm CT, perfusion abnormalities were detected with a high sensitivity and specificity when vessel occlusion was confirmed by criterion standard DSA. No difference was found between lesions sizes measured with the 2 C-arm CT protocols and CTP. Growth of the CBV lesions with time was captured with C-arm CT and CTP.

CONCLUSIONS: In this small study, it was feasible to qualitatively measure CBV and CBF by using a flat panel detector angiographic system.

S troke is a major cause of death and disability throughout the world. IV thrombolysis within 4.5 hours of symptom onset remains the only therapy with benefit for patients so far, as proved by randomized trials. ${ }^{1-3}$ However, the results of studies investigating endovascular approaches indicate that the outcome of patients with large-artery occlusions may be improved in comparison with IV thrombolysis. ${ }^{4-8}$

Perfusion measurements are useful in selecting patients most likely to benefit from revascularization because they provide information about the ratio of infarct core to penumbra. This is especially true for those patients presenting outside the therapeutic time window for IV thrombolysis.

\footnotetext{
Received February 20, 2013; accepted after revision December 19.

From the Institute of Neuroradiology (O.B., C.S., A.L., S.S., M.S.), Department of Neurosurgery (B.J.), and Healthcare Telematics and Medical Engineering (A.B., S.G., G.R.), Otto von Guericke University, Magdeburg, Germany; and Siemens AG (Y.K., Y.D.-Z.), Healthcare Sector, Erlangen, Germany.

This work was supported by the Federal Ministry of Education and Research under grant FKZ 03IP710, and by Siemens AG, Healthcare Sector.

Please address correspondence to Oliver Beuing, MD, Institute of Neuroradiology, Otto von Guericke University Magdeburg, Leipziger Str 44, Magdeburg, 39120, Germany; e-mail: oliver.beuing@med.ovgu.de

- Indicates open access to non-subscribers at www.ajnr.org

http://dx.doi.org/10.3174/ajnr.A3973
}

Despite the potential value of MR imaging or CTP measurements, these studies are often not performed because there is no solid evidence that they improve outcome, there is a significant time cost in obtaining the measurements, and they only provide a snapshot of a dynamic process. ${ }^{9}$ The ability to obtain these measurements in the angiography suite would eliminate the last 2 objections. Several experimental and clinical studies have demonstrated that measurement of CBV is feasible by using C-arm CT. ${ }^{10-12}$ More recently, CBF was measured in healthy swine and canines with such a system. ${ }^{13,14}$ We report our experience using a modified C-arm system to measure steady-state CBV and also to make dynamic measurements of $\mathrm{CBF}$ in an experimental sheep stroke model.

\section{MATERIALS AND METHODS \\ Creation of Stroke}

The experiments were performed in accordance with the guidelines and with written consent of the local ethics authorities. Stroke was successfully created surgically in 6 of 7 German Blackheaded sheep ( 1 animal died during surgery from ventricular fibrillation). For surgery, the animals were placed in a supine position on the angiography table. General anesthesia was inducted with propofol; isoflurane was used for maintenance. Vital parameters were monitored continuously. Surgery was necessary because in sheep, the ICA develops from a rete mirabile, which is 

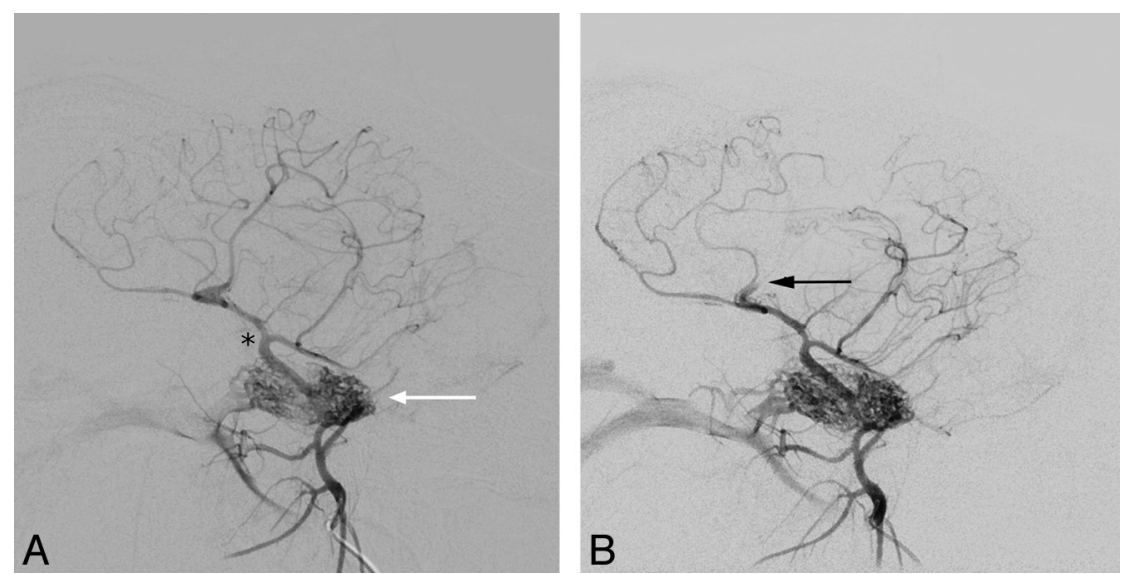

FIG 1. Intracranial vessels before $(A)$ and after $(B)$ vessel occlusion (black arrow). Contrast material was injected near the rete mirabile (white arrow in $A$ ). The MCA surfaces shortly after branching off the ICA (black asterisk in A), which also gives rise to the posterior cerebral artery. Due to early branching, the complete MCA territory was not affected.

formed by extracranial arteries at the skull base (Fig $1 A$ ). The vertebral arteries also join the rete. The intracranial vessels of both sides are connected via communicating arteries. Despite the anatomy, which does not allow direct access to the middle cerebral artery by catheterization, we chose sheep because of their gyrencephalic brain, which is larger than that in other animals used for experimental stroke studies.

The technique for surgical MCA occlusion has been described elsewhere in detail. ${ }^{15}$ In short, trepanation of the skull was followed by incision of the dura mater near where the MCA surfaces. The artery was then occluded by electrocoagulation. Due to anatomic variations in branching of the MCA, the M1 segment could not always be occluded. In 2 cases, only a large M2 branch was occluded. Intra-arterial DSA was performed before surgery to document the vascular anatomy and patency of the intracranial arteries and afterward to confirm artery occlusion (Fig 1B).

\section{Data Acquisition}

$\mathrm{CBV}$ and $\mathrm{CBF}$ perfusion measurements were performed with Carm CT and with a conventional CT scanner. The $\mathrm{C}$-arm of the flat panel angiography system is mounted on a robot arm (Artis zeego; Siemens, Erlangen, Germany), which, due to modifications of the software configuration, allows very fast serial forward and backward rotations, with data acquisition in both directions. With C-arm CT, 2 different protocols were tested. Each comprised 7 rotations (sweeps) of the $\mathrm{C}$-arm before and after contrast administration (baseline and fill runs). The main differences between the 2 protocols lie in the duration of sweeps and the number of projections per rotation. The parameters for the first protocol (slow scan) were as follows: 7 sweeps with a duration of 4.43 seconds each; a 1.57 -second pause between 2 sweeps; rotation angle, $200^{\circ}$; rotation speed, $60^{\circ}$ per second; 248 projections per rotation; angulation step, $0.8^{\circ} /$ frame. The second protocol (fast scan) also comprised 7 sweeps, but with a duration of 2.83 seconds each; a 1.57-second pause between rotations; rotation angle, $200^{\circ}$; rotation speed, $100^{\circ}$ per second; 133 projections per rotation; angulation step, $1.5^{\circ} /$ frame. The $\mathrm{x}$-ray tube voltage was $90 \mathrm{kV}$ in both protocols.

For CTP, a Somatom Emotion 6 (Siemens) was used. The animals were placed on the CT table in a supine position. The head was tilted so that axial images oriented at the frontal skull base by using the topogram could be acquired. To minimize lateral tilting, we adjusted the head by using the laser guide. After contrast injection, the scan was started and the contrast bolus was followed for 60 seconds with 1 acquisition every second.

Identical injection parameters were used for C-arm CT and CTP: IV administration of 42-mL iomeprol (Iomeron 400; Bracco Imaging, Konstanz, Germany) with a power injector (Tennessee; Ulrich Medical, Ulm, Germany) with a flow rate of $6 \mathrm{~mL} / \mathrm{s}$ and an $\mathrm{x}$-ray delay of $12 \mathrm{sec}-$ onds. The injection parameters were determined by testing different injection protocols with varying amounts of contrast media, flow rates, and delays in 2 animals not included in this study. Total scan duration from the start of baseline runs to the end of the measurement amounted to approximately 100 seconds for the slow scan and 75 seconds for the fast scan and CTP, respectively. The radiation dose for the C-arm system was $1.2 \mu$ Gy per projection based on the availability of the hardware and software at that time. Dose-length product was $1047 \mathrm{mGy}-\mathrm{cm}$ for CTP.

Because surgery was performed on the angiography table, imaging with C-arm CT was started immediately after postsurgical DSA. CTP was performed in the adjacent room within 15 minutes after perfusion measurement with C-arm CT. The animal was left in the CT scanner for 3 hours for the second postsurgical measurement and then transported back to the angiography suite. In addition, perfusion imaging with both modalities was performed before vessel occlusion. After completion of the protocols, the animals were euthanized.

\section{Number of Datasets}

Of 84 possible datasets in 7 sheep, only 60 ( 22 before and 38 after surgery) were evaluated (Table 1). Reasons for exclusion of data were death from cardiac failure during surgery $(n=12)$, failed vessel occlusion $(n=8)$, failed contrast injection $(n=2)$, and inability to open the datasets $(n=2)$. Because reconstruction of the data with the prototype software was time-consuming and performed after completion of the protocol, the failed contrast injection and the corruption of datasets were not observed during the course of the procedure. In the case of failed vessel occlusion, DSA could not confirm the success of the surgery. The reason was formation of a blood clot around a catheter that was left in the arteries feeding the rete mirabile for postsurgical DSA. As a result, ipsilateral DSA did not show intracranial vessels and contralateral DSA was not sufficient to depict the vessels on the operated side. Perfusion studies were performed nevertheless because the neurosurgeon was confident that the MCA was occluded.

\section{Postprocessing and Data Analysis}

The CTP data were calculated by using commercially available software (VPCT; Siemens) and also with prototype software 
Table 1: Overview over the acquisition of datasets

\begin{tabular}{|c|c|c|c|c|}
\hline & $\begin{array}{l}\text { C-Arm } 3 \\
\text { Seconds }\end{array}$ & $\begin{array}{l}\text { C-Arm } 5 \\
\text { Seconds }\end{array}$ & $\begin{array}{c}\text { CTP } \\
\text { Prototype }^{a}\end{array}$ & $\begin{array}{c}\text { CTP } \\
\text { VPCT }^{\mathrm{b}}\end{array}$ \\
\hline \multicolumn{5}{|l|}{ Sheep 1} \\
\hline Pre & v & $v$ & v & $v$ \\
\hline 0 Hours & v & v & v & v \\
\hline 3 Hours & v & v & $v$ & $v$ \\
\hline \multicolumn{5}{|l|}{ Sheep 2} \\
\hline Pre & v & $v$ & $v$ & v \\
\hline 0 Hours & v & v & $v$ & v \\
\hline 3 Hours & v & v & v & v \\
\hline \multicolumn{5}{|l|}{ Sheep 3} \\
\hline Pre & v & v & v & $x$ \\
\hline 0 Hours & $x$ & $x$ & v & v \\
\hline 3 Hours & v & v & $v$ & v \\
\hline \multicolumn{5}{|l|}{ Sheep 4} \\
\hline Pre & $x$ & $v$ & $v$ & v \\
\hline 0 Hours & $x$ & $x$ & $x$ & $x$ \\
\hline 3 Hours & $x$ & $x$ & $x$ & $x$ \\
\hline \multirow[t]{3}{*}{ Sheep 5} & $x$ & $x$ & $x$ & $x$ \\
\hline & $x$ & $x$ & $x$ & $x$ \\
\hline & $x$ & $x$ & $x$ & $x$ \\
\hline \multicolumn{5}{|l|}{ Sheep 6} \\
\hline Pre & v & v & v & v \\
\hline 0 Hours & v & v & v & v \\
\hline 3 Hours & v & v & v & v \\
\hline \multicolumn{5}{|l|}{ Sheep 7} \\
\hline Pre & v & $v$ & $v$ & v \\
\hline 0 Hours & v & v & $v$ & v \\
\hline 3 Hours & v & $v$ & $v$ & v \\
\hline Total & 14 & 15 & 16 & 15 \\
\hline
\end{tabular}

Note:-Pre indicates data acquired before surgery; $v$, sets that were acquired as the protocol demanded and that were included in the evaluation; $x$, excluded datasets (The reasons are explained in the text)

a Datasets acquired with CTP and calculated with the prototype software.

b Datasets acquired with CTP and calculated with the commercial software VPCT (Siemens)

developed and provided by Siemens. ${ }^{16}$ This calculation was performed to find out whether the different software implementations would yield similar results with the same data and also to avoid a bias of one algorithm over the other. The data acquired with the C-arm CT were only compatible with the prototype software. So, at every time point (before surgery, immediately and 3 hours after surgery) 4 datasets, each consisting of $1 \mathrm{CBV}$ and 1 CBF color map, were acquired per animal (Fig 2). MTT and TTP were not calculated because the prototype software was not optimized for these parameters.

The arterial input function was chosen manually at the ICA close to the site where it originates from the rete mirabile or the MCA on the side opposite the MCA occlusion (Fig 3). Both the time-attenuation curves and the arterial input functions are undersampled because of the limited temporal resolution (slow rotation) of the C-arm. Specifically, there is only 1 measurement time point per sweep. The intermediate values for all 1-second steps were determined by a bicubic spline interpolation.

Positioning of the head of the animals in the angiographic system was different from that in the CT scanner to allow uninhibited rotation of the $\mathrm{C}$-arm. This required reformatting to generate comparable images concerning section orientation and thickness. Anatomic detail in the perfusion maps acquired was not sufficient to allow automatic registration, so matching of the CT and C-arm images had to be performed on a syngo worksta-

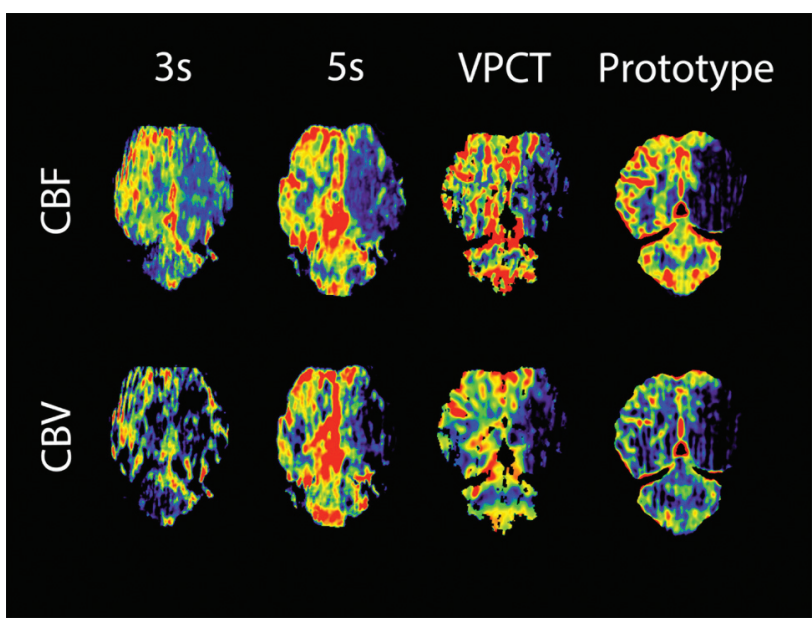

FIG 2. Color maps of CBF and CBV for all protocols 3 hours after vessel occlusion on the left side. Perfusion deficits are clearly visible, but image quality is impaired by streak artifacts, especially in the fast scan. The prototype is CTP data processed with the prototype software. VPCT is CTP data processed with commercial software.

tion (Siemens) on a visual basis by an experienced neuroradiologist who did not take part in the evaluation. The volume datasets acquired with the C-arm system were displayed on the workstation and adjusted by using reference lines, so that the final axial images paralleled the frontal lobe as it did in the CT images. The result of this procedure was directly compared with the timely matching CTP images of the same animal and was further corrected, if necessary, to achieve the best possible agreement.

After anonymization, the color maps were transferred to a certified OsiriX PRO workstation (aycan Digitalsysteme, Würzburg, Germany). They were randomly presented with regard to the time of acquisition and animal subject and were evaluated by 2 experienced neuroradiologists who were blinded to the surgical status. Each neuroradiologist was asked to decide whether perfusion abnormalities were present, and if present, to measure the size in the section exhibiting the largest lesion on a visual basis.

\section{Statistical Analysis}

Interobserver reliability was calculated by using the Cohen $\kappa$ value and was classified according to the Landis and $\operatorname{Koch}^{17}$ scale. Sensitivity and specificity for the detection of perfusion deficits, with DSA-proved vessel occlusion serving as the criterion standard, were determined for each protocol and for both readers. For comparison of the measured sizes of the perfusion lesion, a 2-tailed Student $t$ test was applied to search for differences between the 2 readers. A pair-wise testing was performed to compare the different protocols.

\section{RESULTS}

Interobserver agreement was "substantial" for the fast scan $(\kappa=$ $0.72)$, "almost perfect" for the slow scan $(\kappa=0.86)$, and "perfect" for CTP $(\kappa=1.00)$. Sensitivity and specificity for the detection of perfusion lesions with the different protocols for both readers are outlined in Table 2. With regard to the $\mathrm{C}$-arm protocols, specificity was higher and sensitivity was lower for the fast rather than the slow scans for both readers. For the data obtained with CTP and 

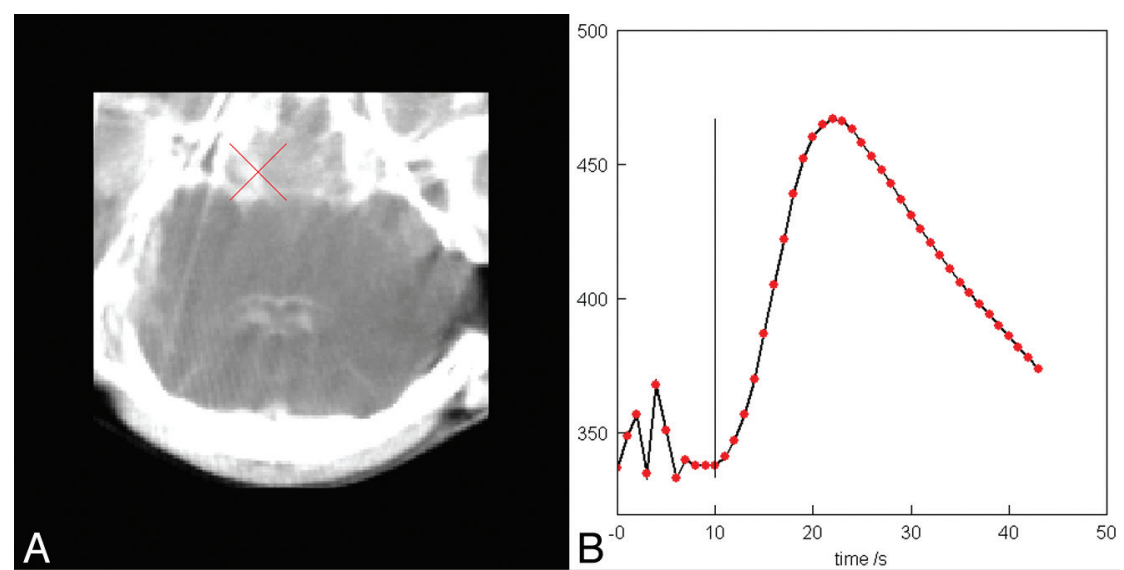

FIG 3. Manually chosen arterial input function in the ICA near the skull base and corresponding time-attenuation curve.

Table 2: Sensitivity and specificity for lesion detection shown separately for each reader and protocol

\begin{tabular}{|c|c|c|c|c|}
\hline & \multicolumn{2}{|c|}{ Reader 1} & \multicolumn{2}{|c|}{ Reader 2} \\
\hline & Occl. & No Occl. & Occl. & No Occl. \\
\hline \multicolumn{5}{|l|}{ Fast } \\
\hline Positive & 8 & 0 & 6 & 0 \\
\hline Negative & 1 & 5 & 3 & 5 \\
\hline Sensitivity & \multicolumn{2}{|c|}{$88.9 \%$} & \multicolumn{2}{|c|}{$66.7 \%$} \\
\hline Specificity & \multicolumn{2}{|c|}{$100 \%$} & \multicolumn{2}{|c|}{$100 \%$} \\
\hline \multicolumn{5}{|l|}{ Slow } \\
\hline Positive & 9 & 1 & 8 & 1 \\
\hline Negative & 0 & 5 & 1 & 5 \\
\hline Sensitivity & \multicolumn{2}{|c|}{$100 \%$} & \multicolumn{2}{|c|}{$88.9 \%$} \\
\hline Specificity & \multicolumn{2}{|c|}{$83.3 \%$} & \multicolumn{2}{|c|}{$83.3 \%$} \\
\hline \multicolumn{5}{|l|}{ Prototype } \\
\hline Positive & 10 & 0 & 10 & 0 \\
\hline Negative & 0 & 6 & 0 & 6 \\
\hline Sensitivity & \multicolumn{2}{|c|}{$100 \%$} & \multicolumn{2}{|c|}{$100 \%$} \\
\hline Specificity & \multicolumn{2}{|c|}{$100 \%$} & \multicolumn{2}{|c|}{$100 \%$} \\
\hline \multicolumn{5}{|l|}{ VPCT } \\
\hline Positive & 10 & 0 & 10 & 0 \\
\hline Negative & 0 & 5 & 0 & 5 \\
\hline Sensitivity & \multicolumn{2}{|c|}{$100 \%$} & \multicolumn{2}{|c|}{$100 \%$} \\
\hline Specificity & \multicolumn{2}{|c|}{$100 \%$} & \multicolumn{2}{|c|}{$100 \%$} \\
\hline
\end{tabular}

Note:-Occl. indicates occlusion.

calculated with the commercial and the prototype software, sensitivity and specificity were $100 \%$.

The average size of the CBF lesions as measured with CTP was $8.3 \pm 3.0 \mathrm{~cm}^{2}$; and for CBV, $7.1 \pm 2.7 \mathrm{~cm}^{2}$. Compared with CTP, the sizes of those lesions that were correctly identified by both readers in the $\mathrm{C}$-arm protocols did not differ significantly in pairwise testing. Reader 2 tended to measure smaller sizes than reader 1 , but this difference was significant only for CBV immediately after surgery $(P=.039)$. CBF lesions were significantly larger than CBV lesions in all protocols $(P=.006$ for the slow scan, for the other protocols $P<.001)$. No significant changes of CBF lesion sizes were noted with time $(P=.33)$. In contrast, CBV lesions were significantly larger 3 hours after vessel occlusion than immediately after surgery $(P=.034)$. Reader 1 correctly identified perfusion deficits in 3 datasets that were not detected by reader 2 . Reader 2 did not detect a lesion that was overlooked by reader 1 .

\section{DISCUSSION}

In ischemic stroke, several factors influence patient outcome. These comprise, among others, time from symptom onset to recanalization, collateral flow, thrombus size, and location of the vessel occlusion. IV thrombolysis is the standard therapy for patients without contraindications and presenting within 4.5 hours after symptom onset, but endovascular therapy may be more effective in patients with occlusion of major cerebral arteries. Additionally, several works indicate that interventional recanalization might be beneficial in patients presenting outside the therapeutic window accepted for IV thrombolysis. ${ }^{18,19}$ However, this relatively new treatment option has drawbacks as well. One is the invasive nature of the procedure with the risk of severe or fatal complications. Consequently, interventional treatment should only be performed in cases with a reasonable chance for improvement of outcome. This alone requires more detailed information regarding the ratio of ischemic core to penumbra than is necessary for initiation of IV thrombolysis. There is evidence that perfusion imaging, though not generally recommended for treatment decisions, serves the purpose of proper patient selection. ${ }^{20-22}$ It might help not only in deciding whether to treat patients but also in refraining from therapy even if the patient presents early after symptom onset. Another drawback of endovascular recanalization is that the time from imaging to treatment start is substantially longer than that for IV thrombolysis. Perfusion imaging is only a snapshot of a dynamic process that cannot predict the speed at which ischemia becomes final infarction. Therefore, saving time is also an essential requirement in optimizing both the efficacy and safety of interventional therapy.

Because performing imaging in the angiographic suite by using the novel C-arm CT addresses both issues, this small study was performed to evaluate whether it is possible, with the described technique, to gain relevant information about brain perfusion with the same device used for therapy, and, consequently, to save time.

The results are encouraging because perfusion lesions were detected in a high or very high percentage with the tested C-arm CT protocols in animals with DSA-proved vessel occlusion. Compared with CTP, the sensitivity was lower for most C-arm CT protocols, but on the other hand, all lesions were small, with the largest matching the size of the human basal ganglia.

The sizes of the perfusion deficits varied considerably among the animals. These differences were due to anatomic variations of the MCA. In some animals, early branching of the MCA was observed and not all branches could be visualized after surfacing. This finding resulted in partial MCA infarction. In other cases, electrocoagulation apparently led to occlusion of the ICA with infarction of the whole anterior circulation territory. Nevertheless, pair-wise testing revealed comparable results with regard to size for the C-arm protocols and CTP. The smallest correctly identified lesion had an area of only approximately $2.5 \mathrm{~cm}^{2}$. That 
reader 2 tended to measure a smaller size for all lesions might be the effect of evaluating datasets of the whole brain. Different sections might have been chosen. However, a more conservative approach to measurement might be taken into account as well.

Size differences between CBF and CBV lesions were measured at both time points, and CBV lesions were larger after 3 hours than immediately after surgery. These size differences of the CBV lesions were significant with all protocols. This finding probably reflects growth of the infarct core with time. That enlargement of CBV lesions found in all protocols and not only in CTP suggests that C-arm CT might be capable of depicting changes of brain perfusion, even if small. This possibility might have an impact on treatment decisions. Perfusion measurement with C-arm CT can be performed not only before therapy but at any time during endovascular stroke intervention. There are thus scenarios in which this information might influence treatment decisions. One such situation could be a difficult intervention with partial recanalization. If no improvement of functional outcome can be expected, the intervention might as well be terminated so that the patient is not exposed to unnecessary risk of complications due to the intervention. In addition, stent placement in the acute phase with all its negative consequences such as dual-antiplatelet therapy might be avoided if perfusion maps indicate a large infarct with little viable parenchyma. Of course, the presented data cannot serve as a basis for validating the use of this technique for such decisions. However, the encouraging results support further effort to reach the goal of making stroke imaging in the angiography suite feasible. C-arm CT already allows the exclusion of intracranial hemorrhage in many cases, and intracranial vessels can be well-depicted. ${ }^{23-26}$ Also, measurement of CBV was feasible by using a C-arm CT. ${ }^{10-12}$ The described technique may additionally provide information about dynamic perfusion parameters.

There are several limitations in this study. First, only a small number of animals were examined. Therefore, the results should be interpreted with caution concerning statistical significance. Nevertheless, a positive trend with regard to lesion detection seems to exist. The complete and irreversible occlusion of a major intracranial artery led to large CBV lesions already in the early phase. Therefore, their enlargement was only slight, and significance might have occurred by chance. Therefore, whether progression of the core infarct can be depicted with C-arm CT remains unclear with this small sample number.

Second, we did not quantify perfusion changes. One reason was that the slow temporal sampling rate leads to an underestimation of the arterial input function; consequently, because CBF values are inversely proportional to the area under the arterial input function, CBF would have been overestimated. ${ }^{27}$ Moreover, our focus was to find out whether assessment of dynamic perfusion parameters was feasible in a new large-animal model. However, quantification is possible as was demonstrated by Ganguly et al. ${ }^{13}$ They stated that CBF measured with C-arm cone beam CT correlated well with that from CTP, but in this work, multiple injections and multiple acquisitions were performed to overcome the temporal resolution limits of the C-arm system, whereas we chose a protocol with a single injection and only 1 fill run.

Third, we chose sheep for our experiments because the technique of surgical occlusion was described and sheep have a larger, gyrencephalic brain, as opposed to that in most other animal stroke models. However, we were not aware that the attenuated bony anatomy of the sheep skull would cause such marked streak artifacts compared with humans. We did not appreciate this drawback during the experiments because postprocessing was performed after having completed the protocol for the animal. When we realized the occurrence of these artifacts, we initially tried to eliminate other possible causes like movement induced by the ventilation tube, exact centering of the brain, or minimizing overlying material needed for anesthesia. The artifacts were more pronounced in the fast scans. Although image quality was not assessed systematically, these artifacts might explain the lower sensitivity compared with the other protocols.

Finally, to ensure the best possible image quality, we chose a high-system x-ray dose. It is likely that new system hardware and advanced software will provide further dose reduction in the future. A dose measurement with an Alderson phantom is being planned.

\section{CONCLUSIONS}

With the described technique, we demonstrated that measurement of CBV and, additionally, $\mathrm{CBF}$ as a dynamic perfusion parameter is feasible by using the novel C-arm system. Information about brain perfusion considered helpful in modern concepts of diagnosis and treatment of acute ischemic stroke may be derived with the same device. This small study supports the effort of further development of a technique that may have the potential to improve management of patients with acute severe stroke by saving time from diagnosis to treatment and by adding information for proper patient selection.

Disclosures: Oliver Beuing—RELATED: Grant: Siemens AG Healthcare Sector, ${ }^{*}$ Federal Ministry of Education and Research,* Comments: The money was used for purchase of the sheep; the facility in which the perfusion studies were performed was supported by the Federal Ministry for Education and Research. Axel BoeseRELATED: Grant: BMBF FKZ 03 IP710 INKA, ${ }^{*}$ Provision of Writing Assistance, Medicines, Equipment, or Administrative Support: Siemens AG, Healthcare Sector, Erlangen, Germany, ${ }^{*}$ Comments: The manufacturer provided sponsored research funding through channels approved by Otto-von-Guericke University; the manufacturer provided equipment for this study and donated all costs associated with the animal study. Cordula Scherlach, Anja Lenz, Steffen Serowy, Georg Rose-RELATED: Grant: Siemens AG, Healthcare Sector, ${ }^{*}$ Federal Ministry of Education and Research.* Sebastien Gugel—RELATED: Support for Travel to Meetings for the Study or Other Purposes: Siemens, ${ }^{*}$ Provision of Writing Assistance, Medicines, Equipment, or Administrative Support: Siemens. ${ }^{*}$ Martin Skalej—RELATED: Grant: Siemens Erlangen, Germany, ${ }^{*}$ Comments: The grant was to pay service costs of a professional animal lab. *Money paid to the institution.

\section{REFERENCES}

1. Tissue plasminogen activator for acute ischemic stroke: the $\mathrm{Na}$ tional Institute of Neurological Disorders and Stroke rt-PA Stroke Study Group. N Engl J Med 1995;333:1581-87

2. Lees KR, Bluhmki E, von Kummer R, et al. Time to treatment with intravenous alteplase and outcome in stroke: an updated pooled analysis of ECASS, ATLANTIS, NINDS, and EPITHET trials. Lancet 2010;375:1695-703

3. Sandercock P, Wardlaw JM, Lindley RI, et al. The benefits and harms of intravenous thrombolysis with recombinant tissue plasminogen activator within $6 \mathrm{~h}$ of acute ischaemic stroke (the third International Stroke Trial [IST-3]): a randomised controlled trial. Lancet 2012;379:2352-63

4. Mattle HP, Arnold M, Georgiadis D, et al. Comparison of intraarte- 
rial and intravenous thrombolysis for ischemic stroke with hyperdense middle cerebral artery sign. Stroke 2008;39:379-83

5. Penumbra Pivotal Stroke Trial Investigators. The Penumbra Pivotal Stroke Trial: safety and effectiveness of a new generation of mechanical devices for clot removal in intracranial large vessel occlusive disease. Stroke 2009;40:2761-68

6. Dávalos A, Pereira VM, Chapot R, et al. Retrospective multicenter study of Solitaire FR for revascularization in the treatment of acute ischemic stroke. Stroke 2012;43:2699-705

7. Nogueira RG, Lutsep HL, Gupta R, et al. Trevo versus Merci retrievers for thrombectomy revascularisation of large vessel occlusions in acute ischaemic stroke (TREVO 2): a randomised trial. Lancet 2012;380:1231-40

8. Saver JL, Jahan R, Levy EI, et al. Solitaire flow restoration device versus the Merci retriever in patients with acute ischaemic stroke (SWIFT): a randomised, parallel-group, non-inferiority trial. Lancet 2012;380:1241-49

9. Lev MH. Perfusion imaging of acute stroke: its role in current and future clinical practice. Radiology 2013;266:22-27

10. Struffert T, Deuerling-Zheng Y, Engelhorn T, et al. Feasibility of cerebral blood volume mapping by flat panel detector $\mathrm{CT}$ in the angiography suite: first experience in patients with acute middle cerebral artery occlusions. AJNR Am J Neuroradiol 2012;33:618-25

11. Struffert T, Deuerling-Zheng Y, Kloska S, et al. Cerebral blood volume imaging by flat detector computed tomography in comparison to conventional multislice perfusion CT. Eur Radiol 2011;21:882-89

12. Bley T, Strother CM, Pulfer K, et al. C-arm CT measurement of cerebral blood volume in ischemic stroke: an experimental study in canines. AJNR Am J Neuroradiol 2010;31:536-40

13. Ganguly A, Fieselmann A, Marks M, et al. Cerebral CT perfusion using an interventional C-arm imaging system: cerebral blood flow measurements. AJNR Am J Neuroradiol 2011;32:1525-31

14. Royalty K, Manhart M, Pulfer K, et al. C-arm CT measurement of cerebral blood volume and cerebral blood flow using a novel highspeed acquisition and a single intravenous contrast injection. AJNR Am J Neuroradiol 2013;34:2131-38

15. Boltze J, Forschler A, Nitzsche B, et al. Permanent middle cerebral artery occlusion in sheep: a novel large animal model of focal cerebral ischemia. J Cereb Blood Flow Metab 2008;28:1951-64
16. Fieselmann A, Dennerlein F, Deuerling-Zheng Y, et al. A model for filtered backprojection reconstruction artifacts due to time-varying attenuation values in perfusion C-arm CT. Phys Med Biol 2011;56:3701-17

17. Landis JR, Koch GG. The measurement of observer agreement for categorical data. Biometrics 1977;33:159-74

18. Abou-Chebl A. Endovascular treatment of acute ischemic stroke may be safely performed with no time window limit in appropriately selected patients. Stroke 2010;41:1996-2000

19. Turk A, Magarik JA, Chaudry I, et al. CT perfusion-guided patient selection for endovascular treatment of acute ischemic stroke is safe and effective. J Neurointerv Surg 2012;4:261-65

20. Bivard A, McElduff P, Spratt N, et al. Defining the extent of irreversible brain ischemia using perfusion computed tomography. Cerebrovasc Dis 2011;31:238-45

21. Davis S, Campbell B, Christensen S, et al. Perfusion/diffusion mismatch is valid and should be used for selecting delayed interventions. Transl Stroke Res 2012;3:188-97

22. Kamalian S, Maas MB, Goldmacher GV, et al. CT cerebral blood flow maps optimally correlate with admission diffusion-weighted imaging in acute stroke but thresholds vary by postprocessing platform. Stroke 2011;42:1923-28

23. Struffert T, Eyupoglu IY, Huttner HB, et al. Clinical evaluation of flat-panel detector compared with multislice computed tomography in 65 patients with acute intracranial hemorrhage: initial results: clinical article. J Neurosurg 2010;113:901-07

24. Blanc R, Pistocchi S, Babic D, et al. Intravenous flat-detector CT angiography in acute ischemic stroke management. Neuroradiology 2012;54:383-91

25. Jeon JS, Sheen SH, Hwang GJ, et al. Feasibility of intravenous flat panel detector CT angiography for intracranial arterial stenosis. AJNR Am J Neuroradiol 2013;34:129-34

26. Doelken M, Struffert T, Richter G, et al. Flat-panel detector volumetric CT for visualization of subarachnoid hemorrhage and ventricles: preliminary results compared to conventional CT. Neuroradiology 2008;50:517-23

27. Konstas AA, Goldmakher GV, Lee TY, et al. Theoretic basis and technical implementations of CT perfusion in acute ischemic stroke. Part 1. Theoretic basis. AJNR Am J Neuroradiol 2009;30:662-68 